\title{
Implementation of Classification based on User using Facebook based Profile
}

\author{
Jeevan Pisal ${ }^{1}$, Nikhil Tanpure ${ }^{1}$, Rahul Hinge ${ }^{1}$, Aniket Potdar ${ }^{1}$, Mrs. Sonali A. Patil ${ }^{2}$ \\ Dept. of Computer Engineering, Bhivarabai Sawant Institute of Technology and Research, Wagholi, Pune, India ${ }^{1}$ \\ Asst. Professor, Dept of Computer Engineering, JSPM's Bhivarabai Sawant Institute of Technology and Research, \\ Wagholi, Pune, India ${ }^{2}$
}

\begin{abstract}
Social Networking Portal like Facebook attracts more and more illegal activities. Social Networking Portal such as Facebook is developed and limitations of Facebook are removed by keeping watch on all types of activities performed on this Portal. Classification of user posts into different categories such as Politics, Education, Entertainment, Sports etc. is made based on analysis of post messages in this Social Network Portal. Interest of any friend can be seen by user of this network from categories graph data. Adult images are blocked into Social Network using adult detection based on Image Processing. User posts, comments are analyzed to classify into Crime, Riots, Worst or Vulgar categories. Sentiment analysis is done on user posts and comments using NLP (Natural Language Processing) algorithm to detect user sentiments. If any user exceeds limit of bad posts or adult images, then he/she is automatically banned from this social networking Portal and this portal is more secure and reliable than Facebook Portal.
\end{abstract}

Keywords: Social Networking, Portal, NLP, Image Processing.

\section{INTRODUCTION}

Social Networking is one of the popular ways to meet people online. It is important for the people using this social networking website to be able to speak their mind without any consequence. Here people typically are seen as mentors to guide the people in a better fashion it's so happens that the people when expressing themself do not think about the feelings of other. They tend to upload images, comments, post which may be volatile in nature and can cause the sentiments of a particular community to be destroyed. In social network like facebook where there are millions of users using facebook to upload and share Varity of pictures. Facebook thereby can't administer or observe these users in dynamic manner, hence they need a system where all the comments pictures are taken and sensed using sentiment analysis.

\section{REVIEW OF RELATED LITERATURE}

We have taken a brief review of all the sigular analysis and related paper to effectively get a preform balance in the implementation.

A. Social Networking

In the recent year social networking has explored in a big way, where multiple parties use social media every single day it has become a paradigm for communication tool and getting people together.

However due to users growing every day it becomes difficult for stopping online bullying, hatred spreading and initiation to groups like IS or ISIS. We need a special program to effectively manage the users via checking the images, post, comments uploaded.

\section{B. Conceptual framework}

This concept was typically based on the experience which was encountered in social networking websites, multiple instances of spreading of hatred, recruitment for terrorist organization is seen here.

\section{IMPLEMENTATION}

We using classification for posts, number of posts are uploaded by user each post is classified in categories like politics, entertainment, sports, education and history. Also each post is analyse and detected either it is bad word post or adult image post or neutral. As per categories of post the graph of posts of user is shown. Only friends of user can see the posts and profile and can chat. If user exceeded the limit of uploading bad word post or adult image post ( in this portal bad word post limit is 4 and adult image post limit is 3) then specific user is automatically banned from this portal. 
Another users can not see the adult image post only warning message goes to the user who posted such type of posts. Comments are also analyse related to post. Graph of posts shows the what type of person he/she is and also shows which type of posts he/she uploaded regularly. In this portal we trying to maintain secure environment.

\section{SYSTEM ARCHITECTURE}

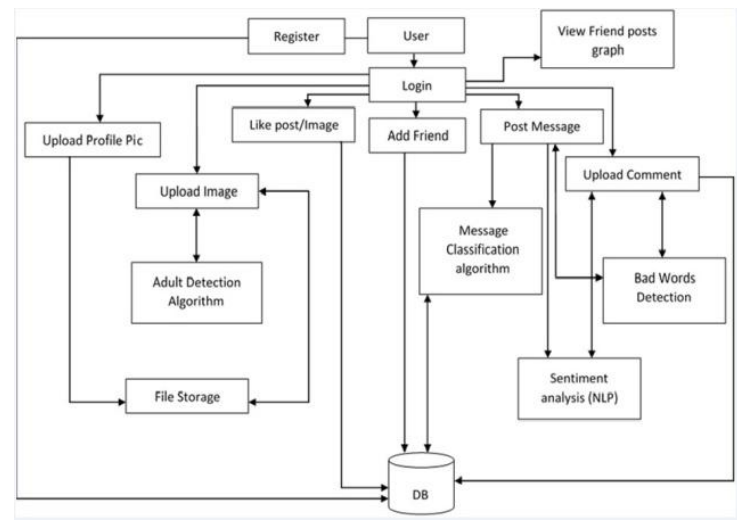

Fig. Architecture of facebook watchdog

\section{SCREENSHOTS}

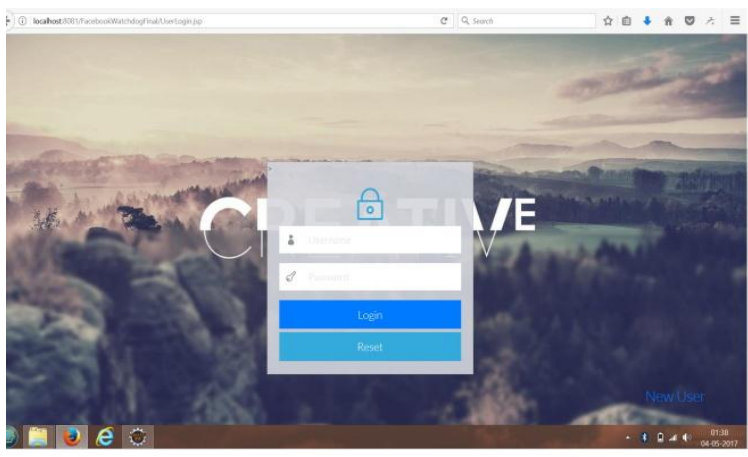

Fig.This screenshot is the home page of application where one can select exiting project

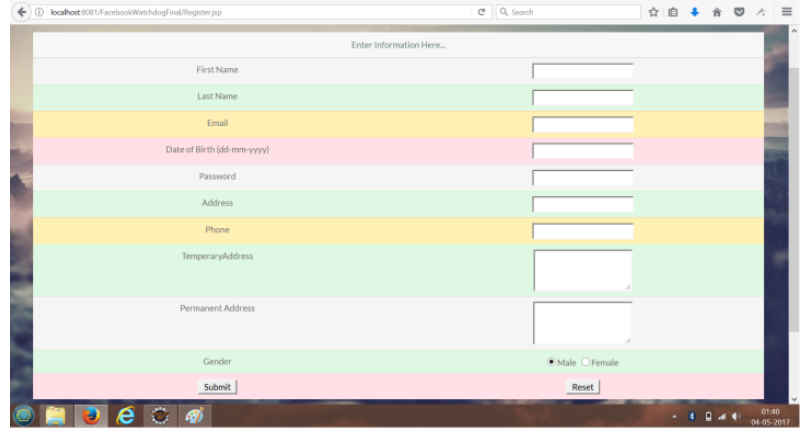

Fig.This screenshot is creating new user profile like creating an account on facebook.

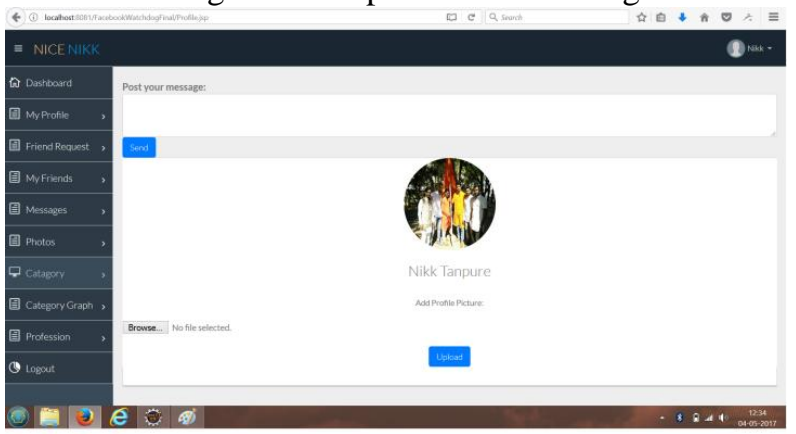

Fig.This screenshot is the profile of the user which is login on the portal. 


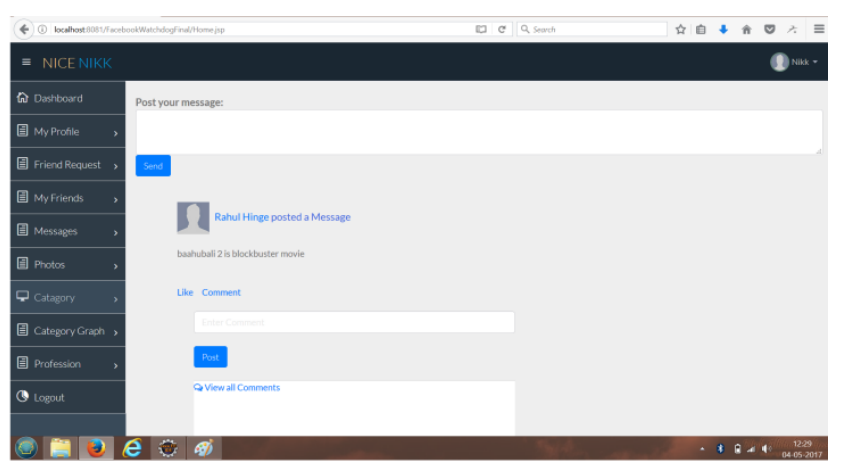

Fig.This screenshot shows the user account activity which contain posting message uploading picture, here we post a message which system categories into different category.

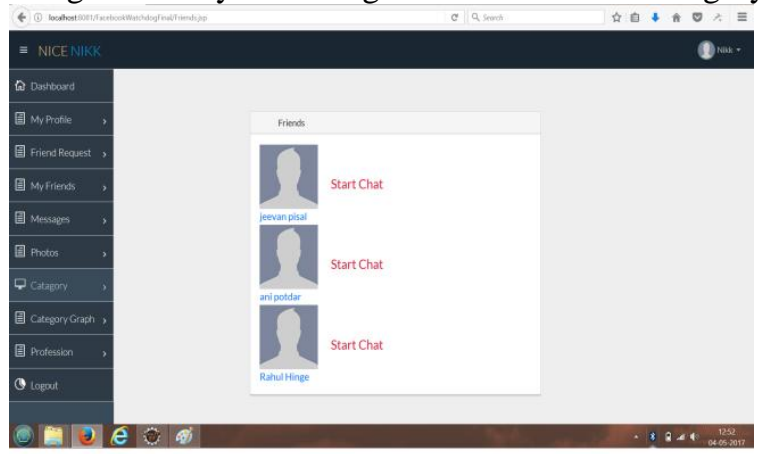

Fig.This screenshot shows the friend list of users account which is login.

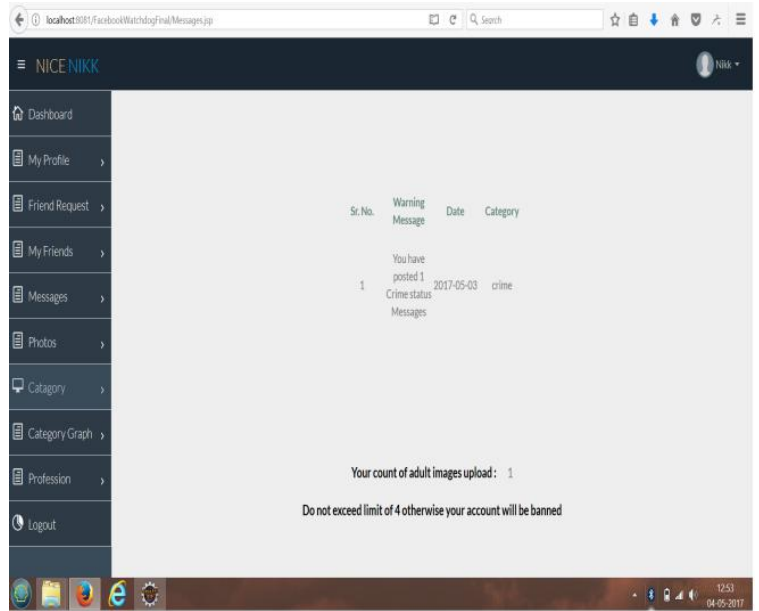

Fig.This screenshot shows how muchtime you upload aAdult images and if you post more than three adult image your account will get block by system. This screen shot also shows how much you post criminal posts.

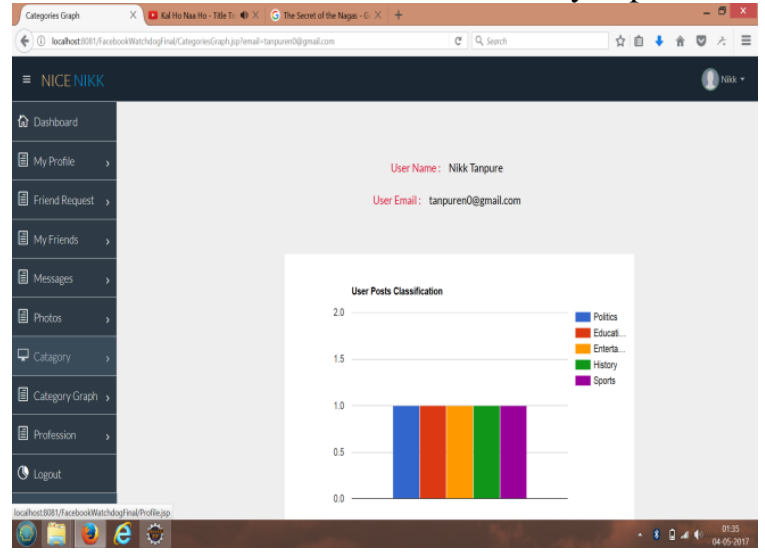

Fig.This screenshot shows a graph which is based on user behavior on the portal. 


\section{CONCLUSION}

In this project Accessing the Facebook post from the developers account. User Posts will be classified into different categories such as Politics, History, Education, etc. Also NLP Algorithm is used for Sentiment Analysis of messages into Positive and Negative Categories.

This System perform to use any user can view other Facebook user's interest based on categories of posts shared by that user such as Politics, History, Education, Entertainment and Sports so that user can get to know about interest of his/her friend.Final output will be on the basis of adult post graph and category of post graph.

\section{ACKNOWLEDGMENT}

This research was partially supported by expert Mr. Akash Bhojraj; we thank him for his help. We thank our colleagues, who provided insight and expertise that greatly assisted the research, although they may not agree with all of the interpretations/conclusions of this paper. We thank our guide for assistance, Mrs. Sonali A. Patil, Professor, JSPM's Bhivrabai Sawant Institute of Technology and Research, Pune for comments that greatly improved the manuscript.

\section{REFERENCES}

[1] Arjumand Younus, Muhammad Atif Qureshi, Josephine Griffith and Colm O'Riordan "“ A Study into the Correlation between Narcissism and Facebook Communication Patterns",IEEE,2015

[2] Marlies Rybnicek, Rainer Poisel and Simon Tjoa, A Research Agenda for detecting online Grooming and bullying activites, 2013

[3] M. Lovelin Ponn Felciah, R.Anbuselvi," A Study on Sentiment Analysis of Social Media Reviews",2015

[4] Sharmishta Desai1, Dr. S.T.Patil2 , Efficient Regression Algorithms for Classification of Social media data,2015

[5] Deepika Sherawat," Mining Emotions (Anger \& fear) from Indian Army Fans Page on Facebook", 2015 